\title{
Camera-projector calibration for near infrared imaging system
}

\author{
M. Yakno1, J. Mohamad-Saleh², M. Z. Ibrahim³, W. N. A. W. Samsudin ${ }^{4}$ \\ ${ }^{1,2}$ School of Electrical and Electronic Engineering, Universiti Sains Malaysia, 14300 Nibong Tebal, \\ Pulau Pinang, Malaysia \\ ${ }^{3,4}$ Faculty of Electrical and Electronics Engineering, Universiti Malaysia Pahang, 26600 Pekan, Pahang, Malaysia
}

\begin{tabular}{|c|c|}
\hline Article Info & ABSTRACT \\
\hline Article history: & \multirow{10}{*}{$\begin{array}{l}\text { Advanced biomedical engineering technologies are continuously changing } \\
\text { the medical practices to improve medical care for patients. Needle insertion } \\
\text { navigation during intravenous catheterization process via Near infrared } \\
\text { (NIR) and camera-projector is one solution. However, the central point of } \\
\text { the problem is the image captured by camera misaligns with the image } \\
\text { projected back on the object of interest. This causes the projected image not } \\
\text { to be overlaid perfectly in the real-world. In this paper, a camera-projector } \\
\text { calibration method is presented. Polynomial algorithm was used to remove } \\
\text { the barrel distortion in captured images. Scaling and translation } \\
\text { transformations are used to correct the geometric distortions introduced } \\
\text { in the image acquisition process. Discrepancies in the captured and projected } \\
\text { images are assessed. The accuracy of the image and the projected image } \\
\text { is 90.643\%. This indicates the feasibility of the captured approach to } \\
\text { eliminate discrepancies in the projection and navigation images. }\end{array}$} \\
\hline Received Aug 2, 2019 & \\
\hline Revised Oct 8, 2019 & \\
\hline Accepted Sep 26, 2019 & \\
\hline Keywords: & \\
\hline Camera-projector calibration & \\
\hline Chessboard patterns & \\
\hline Geometric transformation & \\
\hline Lens distortions & \\
\hline & \\
\hline
\end{tabular}

This is an open access article under the $\underline{C C B Y-S A}$ license.

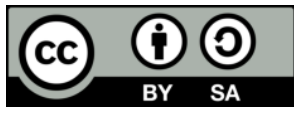

\section{Corresponding Author:}

J. Mohamad-Saleh, School of Electrical and Electronic Engineering,

Universiti Sains Malaysia, 14300 Nibong Teabl, Pulau Pinang, Malaysia.

Email: jms@usm.my

\section{INTRODUCTION}

Intravenous injection is difficult and painful especially for newborns, children, and obese people because appropriate veins maybe deep and not visible. The application of near-infrared light (NIR) has been a good way to help locate vein, since the light can penetrate up to several centimeters into tissues and be absorbed by the vein, which enables discrimination between blood vessels and surrounding tissues [1-4]. Based on the theory, several devices have been developed recently to aid physicians, phlebotomists and surgeons in locating veins to avoid unnecessary sticks.

Some researchers and companies have developed devices which can show an enhanced image on the screen to help locate vein [5-10]. However, it is not convenient for the phlebotomist to watch the screen and do the vein puncture simultaneously. Therefore, the concept of camera-projector system has been introduced to overcome the problem. However, the central point of the image being captured by camera is different than that being projected back. It causes the projected image not to overlay perfectly with the real object. Therefore, it is necessary to calibrate camera and projector.

The first implementation of camera-projector system applied high precision micrometer screw adjusted via mechanical stages to set the optimum position of camera and projector. Then, a simple transformation functions (translation, scale and rotation) was used to align the images on the object [11-12]. Later, was a different method proposed by [13] had improved method developed by [11]. The difference is the placement of the camera, projector and NIR LEDs source in the same axis to remove the hot mirror. 
However, the placement of each component in different locations requires a large space. It also limits the projection area to the region surrounded by the black rectangular plate. In addition, similar back projection method was proposed by [14] using dual camera system. This method was able to obtain the vein depth, but the projection result was similar to single camera system and need huge computation time for the imaging process.

The work by [15] observed that the previously developed projection methods only perform back-projection at a certain distance without observing the results at difference distances. Therefore, [15] proposed a projection method using the intersection between camera view and projection view technique attached with the distance sensor as a parameter to adjust the projection. However, the implementation of the projection method is offline. To be different from others, [16] proposed a combination of NIR imaging technique and augmented reality (AR) technology in head mount device (HMD). This HMD system created a virtual image and overlay onto real-world object to form a composite view in the HMD see-through lenses. However, the adjustment ca mera mount (IR CCD camera) and epipolar geometry are complex and can lead to complex view.

By now, AccuVein AV300 [17] is the most advanced vein display device. However, the structure and the program of the device is intricacy and the cost is very expensive. Therefore, this work proposes to solve the problem of the vein display focusing on optimal camera-projector setting. This paper is structured into five sections. The overview of the camera -projector calibration techniques and the problems encountered are discussed in this section. Section 2 and 3 presents the system structure and the proposed camera-projection calibration. Then the results obtained are presented and discussed in section 4 . Lastly, section 5 concludes the findings of the research work.

\section{SYSTEM STRUCTURE}

This system consists of pico-projector, night vision camera, three sets of 850nm high power NIR LED array, diffuser, based as a target area, raspberry pi $3 \mathrm{~B}+$ and aluminum rod as the main structure. Three high power $3 \mathrm{~W}$ infrared LEDs are focused on the target area and mounted to the left, right and front of the camera. The camera and LEDs are connected to Raspberry Pi with flex cable. The Raspberry Pi and NIR LEDs are derived by a $5 \mathrm{~V} 2.5 \mathrm{~A}$ power supply. The illumination is almost constant during the experiment with the help of a diffuser located in front of each NIR LEDs. The camera is connected to Raspberry pi by way of a short ribbon cable. At another end, the camera is connected to BCM 2837 processor on the PI via the CSI bus.

The pico-projector is placed at the top of the object, at a distance of $45 \mathrm{~cm}$ from the base. The light sources are placed around the camera. The reflected light by NIR LEDs is captured by the cameras on the same side. The distance between the target area and the camera is set to $32 \mathrm{~cm}$. These distances are chosen as the best positions based on a number of trial-and-error experiments to get better overlapping before proceeding on with the lens distortions, scaling and translation. A printed $7 \times 7$ chessboard patterns as a test image is placed on the base. The night version camera is connected to Raspberry Pi board using a CSI cable and pico-projector is connected to the Raspberry Pi using HDMI. After capturing the raw image from the NIR camera module, the images have been processed by a portable Raspberry Pi 3 B+ microcomputer using Open-CV image processing library coded in Python programming language.

\section{CAMERA-PROJECTOR CALIBRATION}

The overall proposed camera-projector calibration procedures are illustrated in Figure 1. It involves three stages; lens distortion correction, scaling transformation and translation transformation. Lens distortion correction has been employed to remove barrel distortion in captured image. Scaling and tra nslation transformations are used to correct geometric distortions introduced in the image acquisition process.

\subsection{Lens distortion correction}

Captured image normally suffered from lens distortion. Based on the literature review by [18-19], the dominant lens distortion is usually barrel distortion. This is due to the imperfection of the lens and the misalignment of the optical elements. Many lens distortion corrections have been proposed from a simple to a complex algorithms. Polynomial algorithm has been used in this work due to its simplicity and accuracy [20-22]. The algorithm starts with $\left(x_{d}, y_{d}\right)$ as the mea sureable coordinates of the distorted image point, $\left(x_{u}, y_{u}\right)$ the coordinates of the undistorted image point. The polynomial algorithm commonly used to describe radialdistortion can be written as [18]:

$$
r_{u}=r_{d}\left(1+k_{1} r_{d}^{2}+k_{2} r_{d}^{4}+\ldots\right)
$$


where $r_{d}$ and $r_{u}$ are the distances of the distorted point $\left(x_{d}, y_{d}\right)$ and the undistorted point $\left(x_{u}, y_{u}\right)$ to the distorted center $P$ respectively, and $k_{i}$ is the parameter of the radialdistortion.

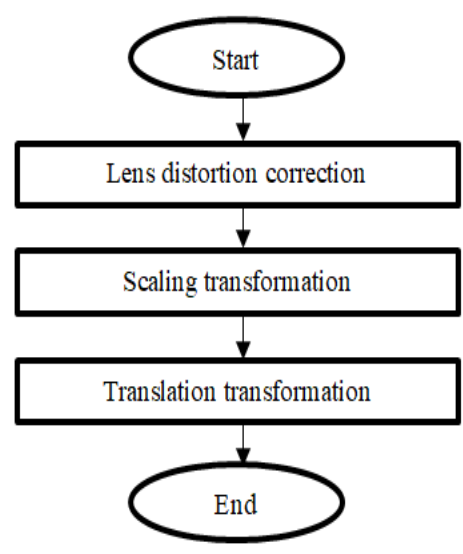

Figure 1. Stages of the proposed camera-projector calibration work

For practical purpose as being explained by [23], only the first term of the infinite series has been used, which is:

$$
r_{u}=r_{d}\left(1+k_{1} r_{d}^{2}\right)
$$

In this work, the contribution of $k$ value towards the appearance of the corrected lens distortion image is investigated. The method of determining the optimum value for $k$ is based on trial-and-error.

\subsection{Scaling transformation}

The problem in this stage is the central point of the image by the camera being different from the one projected back by the projector. Hence, the size of both images normally becomes different. Therefore, image scaling has been applied. Scaling is a linear transformation that is able to enlarge or shrinks' objects by a scale factor that is the same in all directions. An x-value defined the amount of scaling in the $\mathrm{x}$ direction, and a $\mathrm{y}$-value defines the amount of scaling in the $\mathrm{y}$ direction. For each pixel $(x, y)$ of the destination, the source value at the fractional subpixel position is constructed by means of an interpolation object and written to the destination [24]. The coordinates are given by:

$$
\begin{aligned}
& x^{\prime}=\frac{x}{x_{s}} \\
& y^{\prime}=\frac{y}{y_{s}}
\end{aligned}
$$

where $x_{s}$ is the $\mathrm{x}$ scale factor and $y_{s}$ is the y scale factor. When applying scale factors to a source image with width of $w_{s}$ and height of $h_{s}$, the resulting image is defined to have the following dimensions [24]:

$$
\begin{aligned}
& q w_{d}=w_{s} \times x_{s} \\
& h_{d}=h_{s} \times y_{s}
\end{aligned}
$$

Scale factor values greater than 1.0 magnify the image, while value less than 1.0 minify the image. In order to understand the relationship between scale factors and the resultant image, a simple experiment is carried out. The method of obtaining the optimum value for scale factors is based on trial-and-error. Segmented image into three cluster has been used to evaluate the performance of resulted image. The cluster image can be categorized into three regions; black, grey and white pixels. The black pix el represents the real chessboard patterns region, grey pixel represents the projected chessboard region and white pixels represent the background region of the image. From the clustered image, the proposed technique has been quantitatively evaluated by calculating the deviations between the total number of black and grey pixels. The deviations are then calculated as follows:

$$
\text { Deviation }=\frac{\sum \text { black pixels }-\sum \text { grey pixels }}{\sum \text { black pixels }}
$$




\subsection{Translation transformation}

The projected chessboard patterns are not only distorted in terms of size but also the position of the image. Therefore, image translation has been applied after scaling transformation. Image translation is the spatial shifting of an image up, down, left or right. The relationship between the source and destination image coordinates are given by the following equation [25]:

$$
\begin{aligned}
& x_{d}=x_{s}+t_{x} \\
& y_{d}=y_{s}+t_{y}
\end{aligned}
$$

where $x_{d}$ and $y_{d}$ are the integer pixel coordinates of the destination image, $t_{x}$ and $t_{y}$ are the translation values, $x_{s}$ and $y_{s}$ denote the source image point from which the pixel estimate is computed. The projected image also has been converted into three clustered for more precise qualitative and quantitative analysis. The values of $x_{d}$ and $y_{d}$ are determined based on trial-and-error depending upon image. While, for quantitative analysis, is based on overlapping percentages between total number of black and non-overlap grey pixels. The overlapping percentage is calculated using:

$$
\text { overlapping percentages }=\frac{\sum \text { black pixels }-\sum \text { non-overlap grey pixels }}{\sum \text { black pixels }} \times 100
$$

In image acquisition process, the object region of interest is the middle of the chessboard pattern as shown in Figure 2. Therefore, in this work, the best resulted obtained from previous analysis (overlapping percentages) has been divided into three parts; inner, middle and outer parts. The red square represents the inner part, yellow square represents the middle parts and blue square represents the outer parts. The overlapping percentages for these three part are obtained using (10).

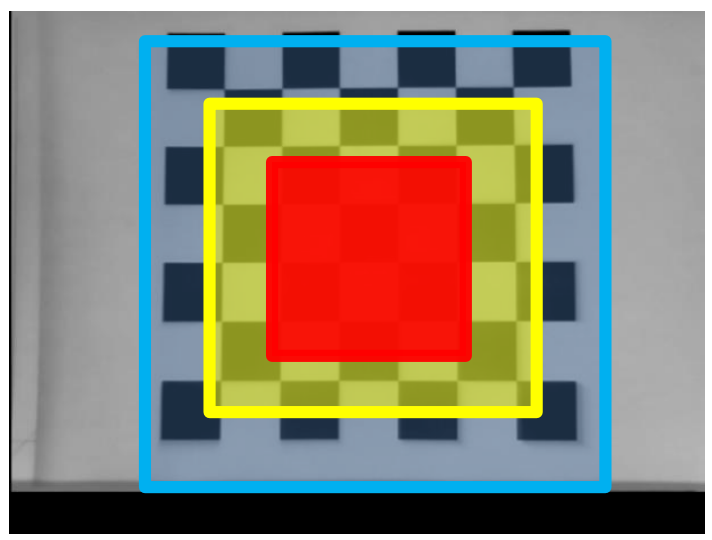

Figure 2. Chessboard patterns with three regions; inner, middle and outer

\section{RESULTS AND DISCUSSION}

Figure 3(a) shows the checkerboard pattern captured using NIR camera and Figure 3(b) is the resultant image projected back onto the real printed chessboard using pico-projector. In Figure 3(b), it can be seen that the projected chessboard pattern is actually the one which looks like a shadow and the black is the real chessboard pattern. This is due to the central point of the image being captured by the camera is different than that being projected back from the projector. As shown in Figure 3 it can be observed that the lines of the chessboard patterns are bulging and its image appears as more magnified at the center than that at the edges due to the barrel distortion.

Figure 4 shows the results of chessboard patterns after lens distortion correction with different values of distortion coefficient, $k$. From the results, it is observed that, before $k=-0.12$ the lines of the chessboard patterns appear straight at the very center of the frame and only start bending away from the center (refer to Figure 4 (b) and (c)). However, after $I=-0.12$, all corrected lines do not go through the center of the image are bowed inwards, towards the center of the image like pincushion (refer to Figure 4 (e) and (f)). Therefore, it can concluded that, the best lens distortion correction image is obviously at $k=-0.12$. 


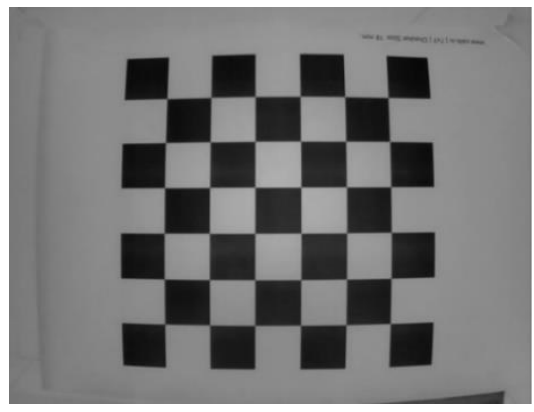

(a)

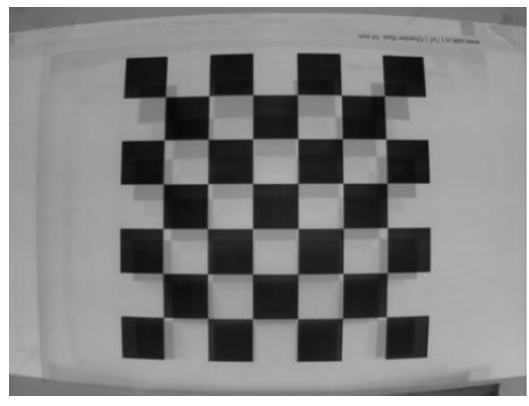

(b)

Figure 3. Image, (a) Of the chessboard pattern taken using NIR source and,(b) Projected back onto real printed chessboard using pico-projector

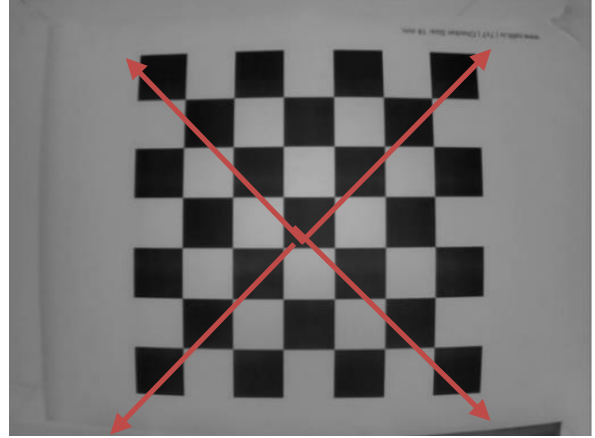

(a)

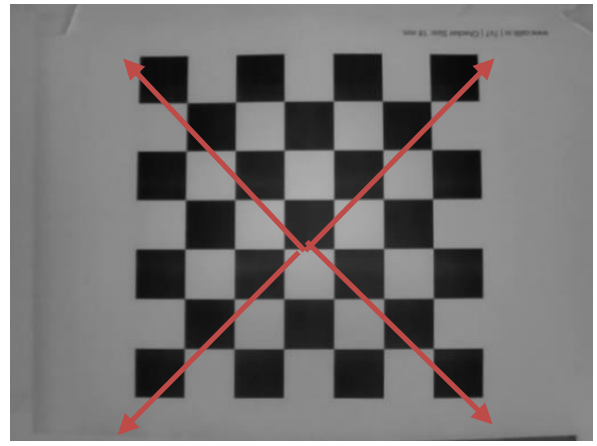

(c)

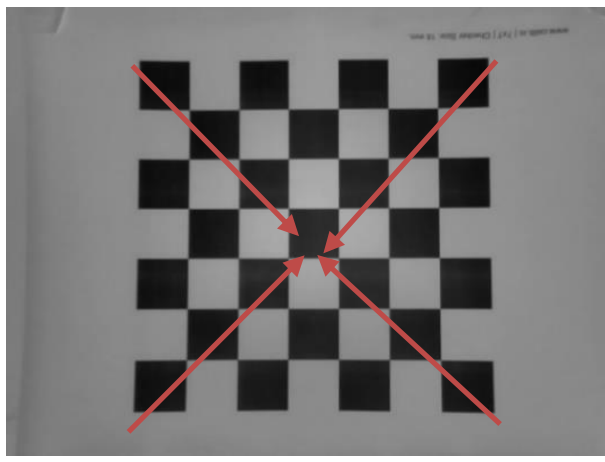

(e)

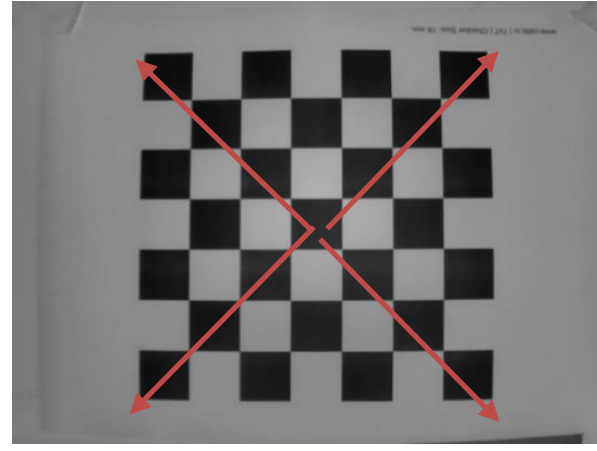

(b)

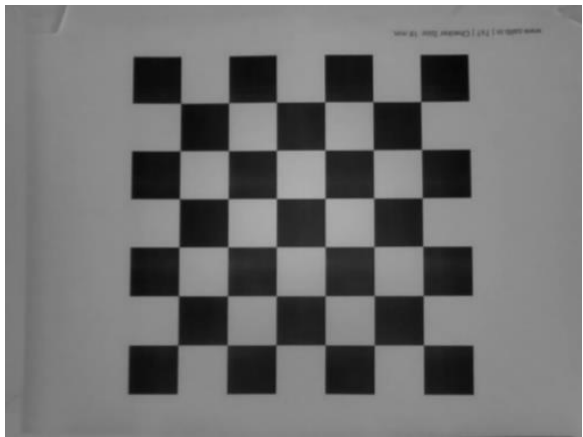

(d)

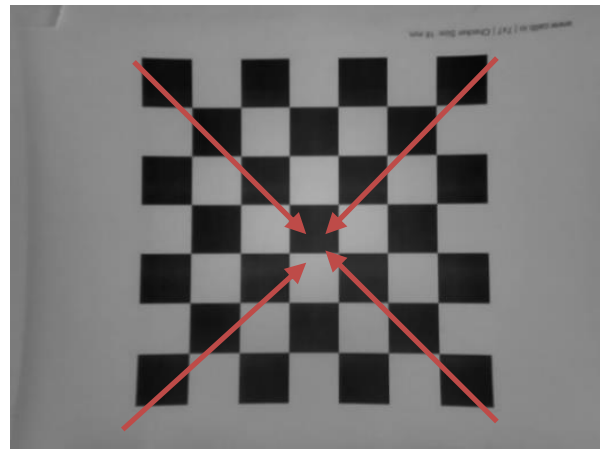

(f)

Figure 4. Results of applying lens distortion correction on, (a) Distorted input image with distortion coefficient of, (b) $k=-0.06$, (c) $k=-0.09$, (d) $k=-0.12$, (e) $k=-0.15$ and (f) $k=-0.18$ 
Table 1 shows the result of scaling the projected chessboard pattern with different values of scaling ratio. The projected image is the one which is in shadow color and the black color is the real chessboard patterns. Cluster image is the images after segmentation process to reduce the difference between real and projected chessboard patterns. As can be seen, at scaling ratio of 1.00 and 1.15, the size of the grey patterns seems significantly small compared to the black patterns. This means that the scaling ratios of 1.00 and 1.15 are not enough to scale-up the size of the projected patterns. Scaling ratio of 1.45 gives bigger size to the projected patterns. Only when scaling ratio is 1.30 , the outputs of the projected and real patterns are almost similar in size. The above results is also supported by deviations between the number of pixels of real patterns and projected patterns as shown in Table 2. The smallest deviation of pixels for both regions is at scaling ratio value of 1.3. Table 3 shows the results of investigation towards determining the optimum values of $x$ and $y$-axes.

Table 1. Projected images of chessboard onto real image patterns with different values of scaling ratio

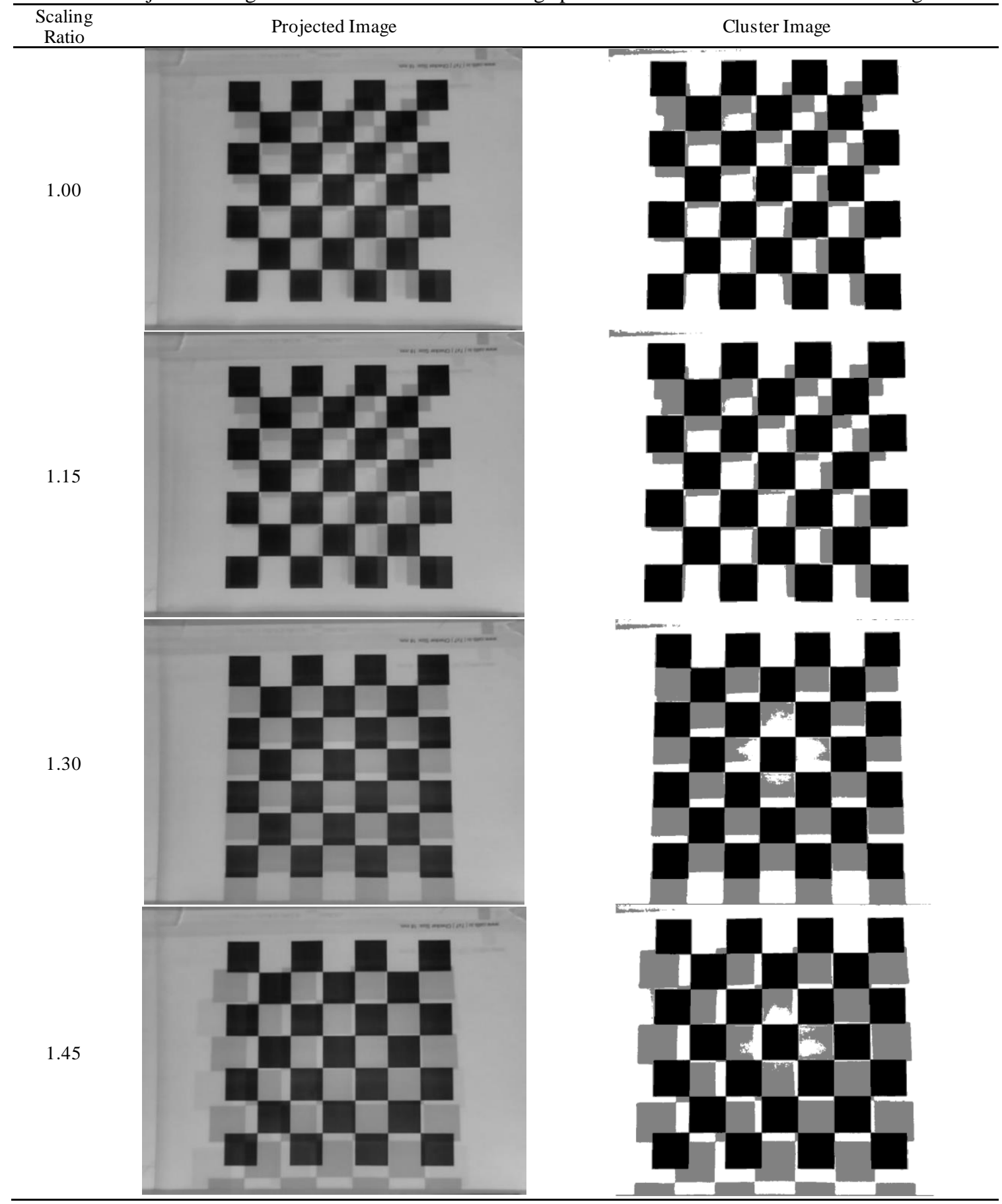


Table 2. Deviation in pixels of scaling ratio between real and projected pixels

\begin{tabular}{cccc}
\hline Scaling & \multicolumn{2}{c}{ Area calculated in pixels } & \multirow{2}{*}{ Deviation in pixels } \\
\cline { 2 - 3 } Ratio & Real ROI (black pixels) & Projected ROI (grey pixels) & \\
\hline 1.00 & 6724 & 5476 & 0.186 \\
1.15 & 6724 & 5625 & 0.163 \\
1.30 & 6241 & 6561 & 0.051 \\
1.45 & 6241 & 7741 & 0.241 \\
\hline
\end{tabular}

Table 3. Results for NIR image translation for different $y$-coordinate values when $x$-axis value is at 3

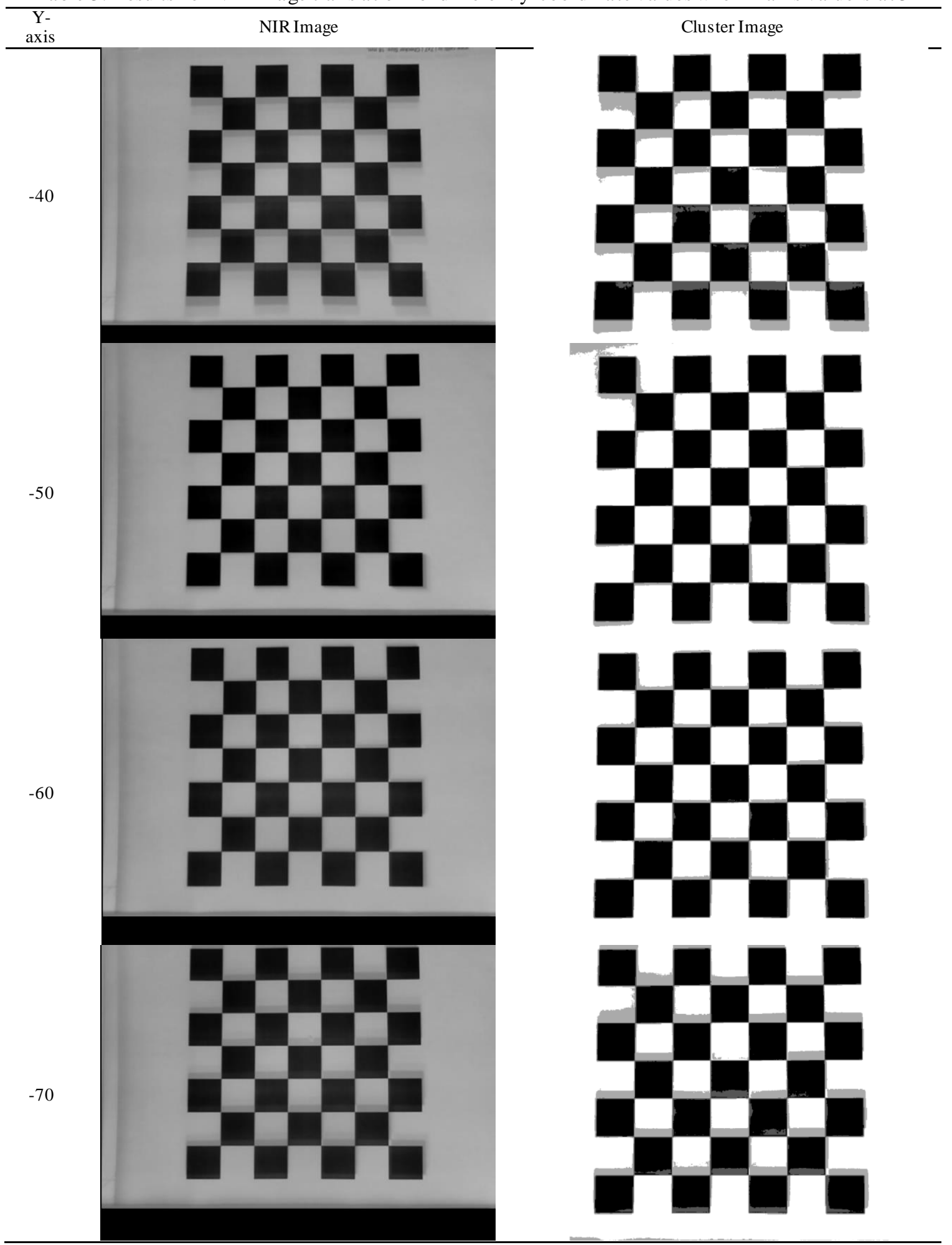

Bulletin of Electr Eng \& Inf, Vol. 9, No. 1, February 2020: 160 - 170 
In order to analyses the results easier, the NIR image has been segmented to cluster image. The cluster image can be categorized into three regions; black, grey and white pixel. The black pixel represents the real chessboard patterns region, grey pixel represents the projected back region and white pixels represented the background region of the image. As can be seen in overall results, when the values of $y$-axis are at -30 and -40 , the projected regions (grey pixels) are located towards the bottom from the real chessboard regions (black pixels). While y-axis is equal to -70 , the projected regions are located towards to the upper of the real chessboard regions. Hence, from the observation, the projected region and real chessboard pattern region almost perfectly overlap to each other when $\mathrm{x}$-axis equals to 3 and $\mathrm{y}$-axis equals to -50 compared to other results. This results are also supported by the overlapping percentages as shown in Table 4 . The $x$-axis $=3$ and $y$-axis $=-50$ produced the highest overlapping percentages of $90.643 \%$. This shows that the values of $\mathrm{x}$ and $\mathrm{y}$ axes at that point is the most optimum for translation transformation. Next, the optimum translation values of $\mathrm{x}$ and $\mathrm{y}$ axes can be analyzed more details by dividing the cluster image into 3 regions; inner, middle and outer. This is due to the future process, where the ROI will be focused on the center of the cluster image, which is the inner and middle regions.

Table 4. Overlapping percentages for each values of $\mathrm{x}$ and $\mathrm{y}$-axes translation

\begin{tabular}{ccccc}
\hline X-axis & Y-axis & $\begin{array}{c}\text { Total number of real pixels } \\
\text { (black) }\end{array}$ & $\begin{array}{c}\text { Total number of non-overlap } \\
\text { projected pixels (grey) }\end{array}$ & \begin{tabular}{c} 
Overlapping percentages $(\%)$ \\
\hline
\end{tabular} \\
\hline & -30 & 129346 & 54073 & 58.195 \\
& -40 & 129407 & 41024 & 68.298 \\
& -50 & 129586 & 25910 & 80.006 \\
& -60 & 129810 & 26428 & 89.641 \\
& -70 & 129510 & 35788 & 72.367 \\
0 & -30 & 129231 & 51676 & 60.013 \\
& -40 & 129431 & 37973 & 70.662 \\
& -50 & 129396 & 20670 & 85.026 \\
& -60 & 129612 & 19376 & 72.492 \\
& -70 & 129483 & 35618 & 58.394 \\
3 & -30 & 128986 & 53666 & 70.744 \\
& -40 & 129193 & 37797 & 90.643 \\
& -50 & 129090 & 12079 & 86.531 \\
& -60 & 129498 & 17442 & 72.723 \\
6 & -70 & 129383 & 35292 & 59.294 \\
6 & -30 & 128820 & 52437 & 70.666 \\
& -40 & 128921 & 37818 & 82.035 \\
& -50 & 129209 & 24505 & 71.035 \\
& -60 & 129375 & 22138 & 57.243 \\
9 & -70 & 129421 & 37487 & 68.235 \\
& -30 & 128701 & 55029 & 78.448 \\
& -40 & 129071 & 41000 & 79.644 \\
& -50 & 129171 & 27839 & 70.071 \\
\hline
\end{tabular}

Table 5 shows the overlapping percentages for inner, middle and outer regions of the chessboard patterns at optimum $\mathrm{x}$ and $\mathrm{y}$-axes translation. As can be seen, the inner region produced the highest overlapping percentage, $92.915 \%$ followed by middle region with $90.925 \%$. Although the outer region is not the focus of this work, its overlapping percentage also approaches to $90 \%$. Figure 5 shows the results of test objects projected onto the chessboard patterns. As can be seen, the test objects have been successfully projected on and aligned.

Table 5. Overlapping percentages for inner, middle and outer regions of chessboard patterns at translation $\mathrm{x}=3$ and $\mathrm{y}=-50$

\begin{tabular}{cccc}
\hline \multirow{2}{*}{ Region } & \multicolumn{2}{c}{ Areas calculated in pixels } & \multirow{2}{*}{ Overlapping percentage } \\
\hline Inner & Black pixels & Non-overlap grey Pixels & \\
Middle & 24968 & 1769 & 92.915 \\
Outer & 41994 & 3811 & 90.925 \\
\hline
\end{tabular}




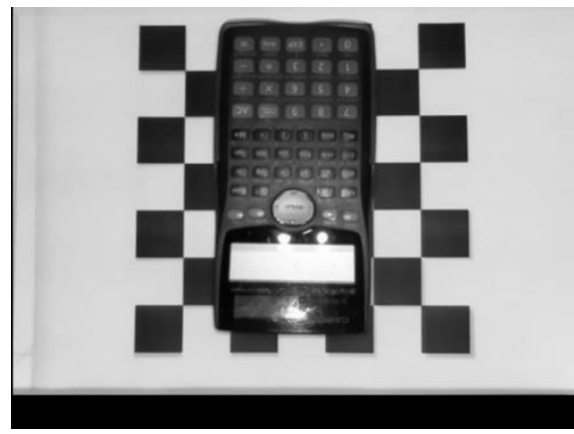

(a)

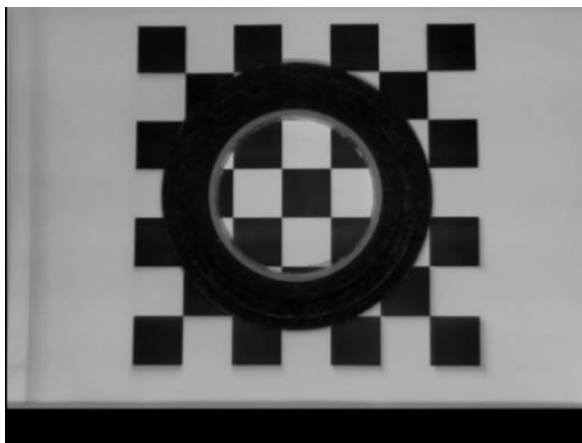

(c)

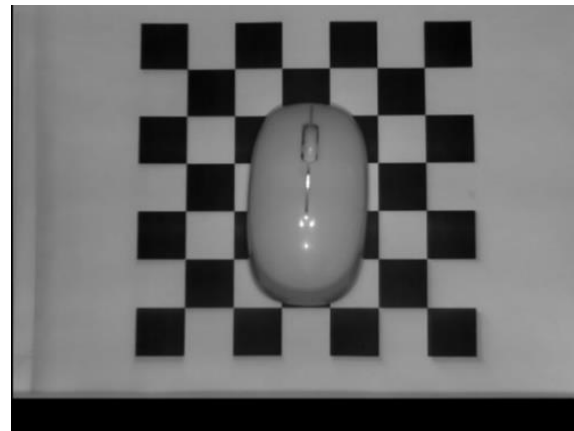

(b)

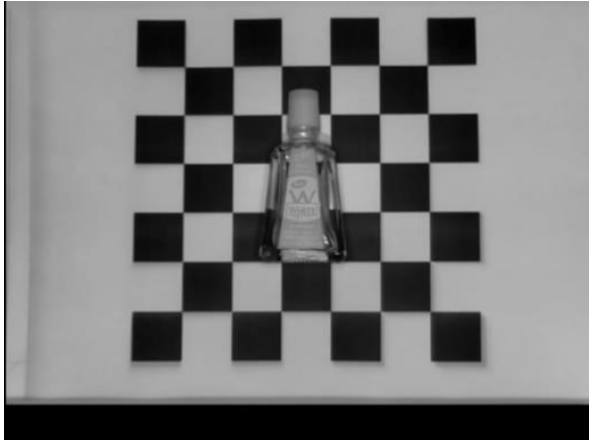

(d)

Figure 5. A real test objects to verified the successful camera-projector calibration and a lignment

\section{CONCLUSION}

This paper presented method to calibrate a camera-projector system to be used in a vein visualization system without any high precision mechanical parts. First, the camera-projector system was applied with lens distortion correction to remove the barrel distortion in captured image by using the simplest technique of polynomial algorithm. Then, scaling and translation transformation were used to correct geometric distortions, so that the projected image is overlaid onto the exact location of object in the real-world. This method is formed fea sible and able to solve the camera-projectorcalibration problem.

\section{ACKNOWLEDGEMENTS}

The authors acknowledge the Universiti Malaysia Pahang internal grant: RDU 1703225 for the financialsupport.

\section{REFERENCES}

[1] E. M. A. E. Subha Fairuz, Mohamed Hadi Habaebi, "Pre-trained Based CNN Model to Identify Finger Vein," Bulletin of Electrical Engineering and Informatics, vol. 8, no. 3, pp. 855-862, 2019.

[2] K. I. Ahmed, M. H. Habaebi, and M. R. Islam, "A real time vein detection system," Indones. J. Electr. Eng. Comput. Sci., vol. 10, no. 1, pp. 129-137, 2018.

[3] C. A. Mela, D. P. Lemmer, F. S. Bao, F. Papay, T. Hicks, and Y. Liu, "Real-time dual-modal vein imaging system," Int. J. Comput. Assist. Radiol. Surg, vol.14. no. 2, pp. 203-213, Oct. 2019.

[4] S. Crisan and B. Tebrean, "Low cost, high quality vein pattern recognition device with liveness Detection. Workflow and implementations," Meas. J. Int. Meas. Confed., vol. 108, pp. 207-216, 2017.

[5] K. I. Ahmed, M. H. Habaebi, and M. R. Islam, "Blood vein detection system for smartphones," in International Conference on Computer and Communication Engineering, pp. 459-464, 2018.

[6] M. Z. Yildiz and Ö. F. Boyraz, "Development of a low-cost microcomputer based vein imaging system," Infrared Phys. Technol., vol. 98, no. February, vol. 98, pp. 27-35, 2019.

[7] M. R. I. Kazi Istiaque Ahmed, Mohamed Hadi Habaebi, "Smartphone Aided Real-Time Blood Vein Detection System," Bulletin of Electrical Engineering and Informatics , vol. 8, no. 3, pp. 1096-1107, 2019. 
[8] A. M. R. R. Bandara, K. A. S. H. K. Rajarata, and P. W. G. R. M. P. B. Giragama, "Super-efficient spatially adaptive contrast enhancement algorithm for superficial vein imaging," 2017 IEEE Int. Conf. Ind. Inf. Syst. ICIIS 2017 - Proc., pp. 1-6, 2017.

[9] D. Kim, Y. Kim, S. Yoon, and D. Lee, "Preliminary study for designing a novel vein-visualizing device," Sensors (Switzerland), vol. 17, no. 2, 2017.

[10] G. Cantor-peled, Ovadia-Blechman, and M. H. Zehava, "Peripheral vein locating techniques," Imaging Med, vol. 8, no. 3, pp. 83-88, 2016.

[11] H. D. Zeman, "Prototype vein contrast enhancer," Opt. Eng., vol. 44, no. 8, pp. 086401, 2005.

[12] H. D. Zeman, G. Lovhoiden and C. Vrancken, "The clinical evaluation of vein contrast enhancement," The 26th Annual International Conference of the IEEE Engineering in Medicine and Biology Society, San Francisco, CA, pp. 1203-1206, 2004

[13] X. Dai, Y. Zhou, X. Hu, M. Liu, X. Zhu and Z. Wu, "A fast vein display device based on the camera-projector system," 2013 IEEE International Conference on Imaging Systems and Techniques (IST), Beijing, pp. 146-149, 2013.

[14] D. Ai et al., "Augmented reality based real-time subcutaneous vein imaging system," Biomed. Opt. Express, vol. 7, no. 7, pp. 2565, 2016.

[15] I. P. A. S. Gunawan, R. Sigit, and A. I. Gunawan, "Vein Visualization System Using Camera and Projector Based on Distance Sensor," 2018 Int. Electron. Symp. Eng. Technol. Appl. IES-ETA 2018 - Proc., pp. 150-156, 2018.

[16] G. C. Meng, A. Shahzad, N. M. Saad, A. S. Malik and F. Meriaudeau, "Prototype design for wearable veins localization system using near infrared imaging technique," 2015 IEEE 11th International Colloquium on Signal Processing \& Its Applications (CSPA), Kuala Lumpur, pp. 112-115, 2015.

[17] J. C. De Graaff, N. J. Cuper, R. A. A. Mungra, K. Vlaardingerbroek, S. C. Numan, and C. J. Kalkman, "Near-infrared light to aid peripheral intravenous cannulation in children: A cluster randomised clinical trial of three devices," Anaesthesia, vol. 68, no. 8, pp. 835-845, 2013.

[18] P. Drap and J. Lefevre, "An exact formula for calculating inverse radial lens distortions," Sensors, vol. 16, no. 6, pp. 1-18, 2016.

[19] X. Y. B, X. Mei, S. Yang, G. Wang, and J. Rong, "Imposing differential constaints on radial distortion correction," Lect. Notes Springer Int. Publ., vol. 9004, pp. 384-398, 2015.

[20] D. Santana-Cedrés et al., "An Iterative Optimization Algorithm for Lens Distortion Correction Using Two-Parameter Models," Image Process. Line, vol. 6, pp. 326-364, 2016.

[21] X. Li, X. Su, Z. Hu, L. Yang, L. Zhang, and F. Chen, "Improved distortion correction method and applications for large aperture infrared tracking cameras," Infrared Phys. Technol., vol. 98, pp. 82-88, 2019.

[22] Z. Tang, R. Grompone von Gioi, P. Monasse and J. Morel, "A Precision Analysis of Camera Distortion Models," in IEEE Transactions on Image Processing, vol. 26, no. 6, pp. 2694-2704, June 2017.

[23] Q. Sun et al., "Camera self-calibration with lens distortion,” Optik (Stuttg)., vol. 127, no. 10, pp. 4506-4513, 2016.

[24] A. J. R. Joe and R. N, "Scaling Transform Methods For Compressing a 2D Graphical image," Adv. Comput. An Int. J., vol. 4, no. 2, pp. 41-50, 2013.

[25] Y. Zhang, "Image processing using spatial transform," Proc. 2009 Int. Conf. Image Anal. Signal Process. IASP 2009, vol. 2, no. 1, pp. 282-285, 2009.

\section{BIOGRAPHIES OF AUTHORS}

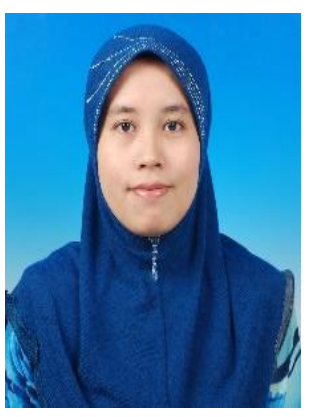

Marlina Yakno received her BEng in electrical and electronic engineering from the Universiti Tenaga Nasional, Malaysia in 2007 and MSc from the Universiti Sains Malaysia in 2013. She is a lecturer in the Universiti Malaysia Pahang and currently pursuing her PhD at the School of Electrical and Electronic Engineering, Universiti Sains Malaysia. Her research interests include image processing, computer vision, embedded system and computational intelligence.

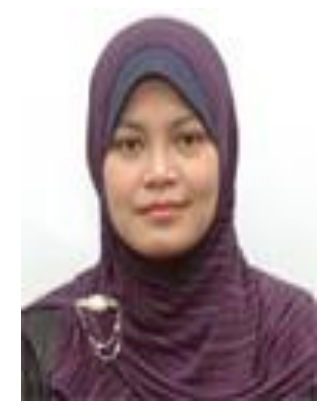

Junita Mohamad-Saleh received her BSc (in computer engineering) degree from the Case Western Reserve University, USA in 1994, MSc from the University of Sheffield, UK in 1996, and PhD from the University of Leeds, UK in 2002. She is currently an Associate Professor in the School of Electrical and Electronic Engineering, Universiti Sains Malaysia. Her research interests include computational intelligence, tomographic imaging, and parallel processing. 

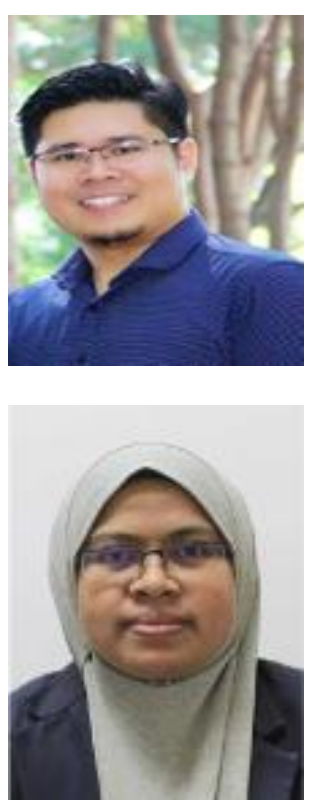

Mohd Zamri Ibrahim received her BEng and MEng in electrical engineering from the Universiti Teknologi Malaysia, Malaysia in 2004 and 2008 respectively. He earns his $\mathrm{PhD}$ from Loughborough University, UK in 2014. He is currently a senior lecturer in the Faculty of Electrical and Electronics Engineering, Universiti Malaysia Pahang. His research interests include computer vision, plasma science, embedded system programming, brain computer interaction, image processing, intelligent system, deep learning and speech recognition.

Wan Nur Azhani W. Samsudin received her BEng in Electrical and Electronic Engineering from the Universiti Malaysia Pahang, Malaysia in 2007 and Msc, majoring in Computer Science from Universiti Malaya, Malaysia in 2010. She earns her PhD from Universiti Malaysia Pahang in Electrical Engineering in 2017 and currently a senior lecturer in the Faculty of Electrical and Electronic Engineering, Universiti Malaysia Pahang. Her research interest is in computer and machine vision, image processing, deep learning and surveillance system. 